\title{
Electrical Characteristics and Temperature Dependence of Photovoltaic Parameters of GaInAsSb Based TPV Diode
}

\author{
B. KucuR ${ }^{a, *}$, M. Ahmetoglu ${ }^{a}$, I.A. Andreev ${ }^{b}$, E.V. Kunitsyna ${ }^{b}$, M.P. Mikhailova \\ AND Y.P. YAKOVLEV ${ }^{b}$ \\ ${ }^{a}$ Department of Physics, Faculty of Sciences and Arts, Uludag University, 16059, Bursa, Turkey \\ ${ }^{b}$ Ioffe Physical-Technical Institute, Russian Academy of Sciences, Saint Petersburg 194021, Russia \\ In this paper, electrical characterization of low bandgap GaInAsSb based thermophotovoltaic (TPV) diodes \\ were investigated, as well as the temperature dependence of photovoltaic parameters such as short circuit cur- \\ rent $\left(I_{\mathrm{sc}}\right)$ and open circuit voltage $\left(V_{\mathrm{oc}}\right)$. Investigation of the dark current mechanisms of the structure was carried \\ out at several temperatures. The effect of light intensity on current-voltage characteristics was also investigated.
}

DOI: 10.12693/APhysPolA.129.767

PACS/topics: 73.40.Lq, 72.40.+w, 85.60.- $\mathrm{q}$

\section{Introduction}

Thermophotovoltaic (TPV) structures convert infrared radiation emitted from a heated source directly into electricity via photovoltaic effect. For almost 50 years, TPV converters have attracted interest of researchers but the real improvement has been seen only in the past two decades $[1,2]$. There have been many attempts to increase the efficiency of these systems [3]. Low band-gap energies make GaSb, InAs and related compounds promising candidates for thermophotovoltaic applications [4], as well as for photodiodes, light emitting diodes, infrared lasers, solar cells, etc. [5-7]. For high efficiency, it is important for the TPV structure to have a closer bandgap energy to the energy of the incident photon. Quaternary GaInAsSb alloys can be grown lattice matched to GaSb substrates and variation of the composition of the alloy can alter the bandgap of the structure $[8,9]$. To date, GaSb-based single junction TPV cells have been widely used for TPV generators [10].

We have reported the characterization of the structures of different type and of small diameter in our previous work $[4,11]$. In this study, we have focused on $\mathrm{p}-\mathrm{GaSb} / \mathrm{p}-$ GaInAsSb/n-GaAlAsSb thermophotovoltaic diode operating in the mid-infrared spectral range. We have investigated electrical and optical properties of the structure, in order to have a better knowledge about the behaviour of the structure for higher performance. The aim of this study is to provide contribution to the improvement of TPV structures.

\section{Experimental}

The p-GaSb/p-GaInAsSb/n-GaAlAsSb thermophotovoltaic diode was fabricated by liquid-phase epitaxy (LPE) at Ioffe Physicotechnical Institute. High quality

*corresponding author; e-mail: banukucur@uludag.edu.tr
$\mathrm{GaIn}_{0.22} \mathrm{AsSb}$ solid solution with composition near the miscibility gap boundary at $T=600^{\circ} \mathrm{C}$ was grown on lattice matched $\mathrm{p}$-GaSb substrate. According to X-ray diffractometry data, the lattice mismatch between the epitaxial layer and GaSb substrate was positive $(\Delta a / a=$ $(2-5) \times 10^{-4}$ at room temperature). Wide bandgap GaAlAsSb solid solution was used as wide-gap window for GaInAsSb-based structure, to lower the surface recombination velocity [12].

Current-voltage characteristics were measured using a KEITHLEY 6517A Electrometer. All measurements were controlled by a computer via an IEEE-488 standard interface, so that data collecting, processing and plotting could be accomplished automatically. Fabricated devices were mounted into a glass dewar with a cold shield for detailed electrical measurements at various temperatures. Photovoltaic measurements were employed using a $150 \mathrm{~W}$ halogen lamp and THORLABS FES1000 Infrared Filter. The intensity of halogen lamp radiation was varied by changing the distance of the sample from the source. The intensity of the incident light was measured by THORLABS Photo Diode Amplifier PDA200C with DET36A Si Biased Detector and DET10D Extended InGaAs Biased Detector.

\section{Results and discussion}

Forward and reverse current-voltage $(I-V)$ characteristics of the GaSb/GaInAsSb/GaAlAsSb thermophotovoltaic diode at different temperatures have been investigated. Figure 1 shows dark $I-V$ characteristic of the structure with a $1 \mathrm{~mm}$ active region diameter. Forward current mechanism was determined to be the diffusion and generation-recombination (g-r) mechanisms of current transport at temperatures in the ranges from $345 \mathrm{~K}$ to $310 \mathrm{~K}$ and from $300 \mathrm{~K}$ to $250 \mathrm{~K}$, with the ideality factor $(\beta)$ values changing from 1.04 to 2.3 , respectively (inset of Fig. 1). At low temperatures the tunneling mechanism became dominant with a higher $\beta$ value. The weak dependence of current on temperature can be considered as an evidence for this assumption [13]. 


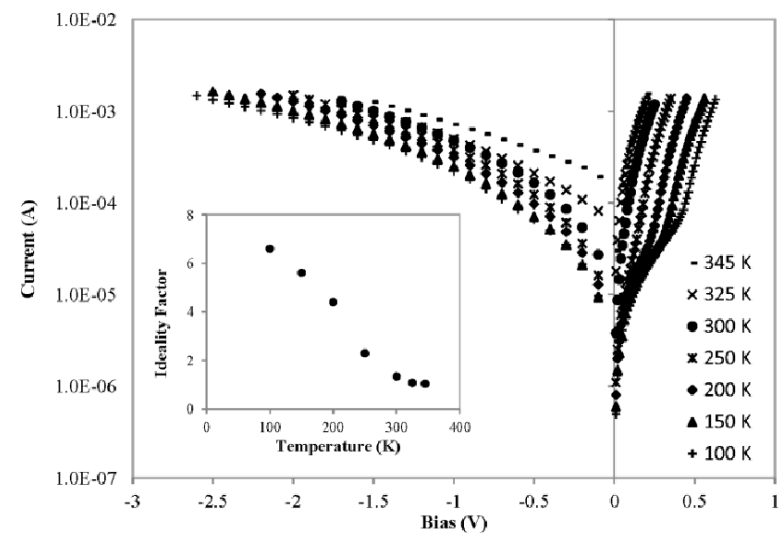

Fig. 1. $I-V$ characteristics of the structure at several temperatures. In the inset the ideality factor values are shown.

Figure 2 presents the exponential dark current values as a function of reciprocal temperature for different biases. The activation energy derived from this dependence over the range from $345 \mathrm{~K}$ to $300 \mathrm{~K}$ is $E_{a}=0.23 \pm 0.02 \mathrm{eV}$ at $-1.0 \mathrm{~V}$, which is close to the half of the bandgap width for the GaInAsSb solid solution used in the active region of the heterostructure. This is the evidence for the generation mechanism of the flow of the dark current.

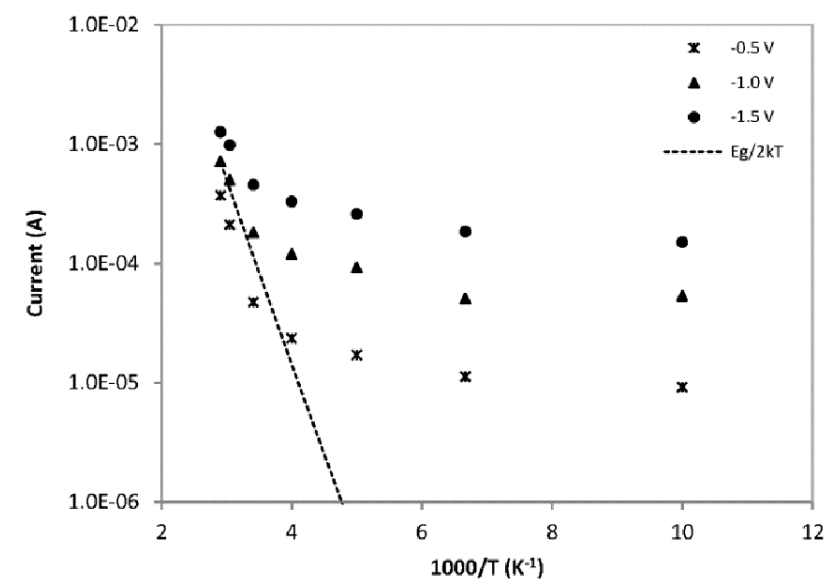

Fig. 2. The reverse current as a function of reciprocal temperature at different reverse biases.

The experimental data in this temperature range agree with the calculated dependence for the g-r current, which has a temperature dependence of the form $I \propto$ $T^{3 / 2} \exp \left(-E_{\mathrm{g}} / 2 k T\right)$ [13]. The tunneling mechanism is temperature independent. The deviation from the theoretical values is due to the increasing tunneling component of the dark current at low temperatures and/or large reverse bias voltages [14].

For optical characterization the $I-V$ measurements were performed under different illumination intensities (Fig. 3). Quartz halogen tungsten lamp was used as a light source with the THORLABS FES1000 infrared filter. The intensity was varied by mounting the structure at different distances from the source.

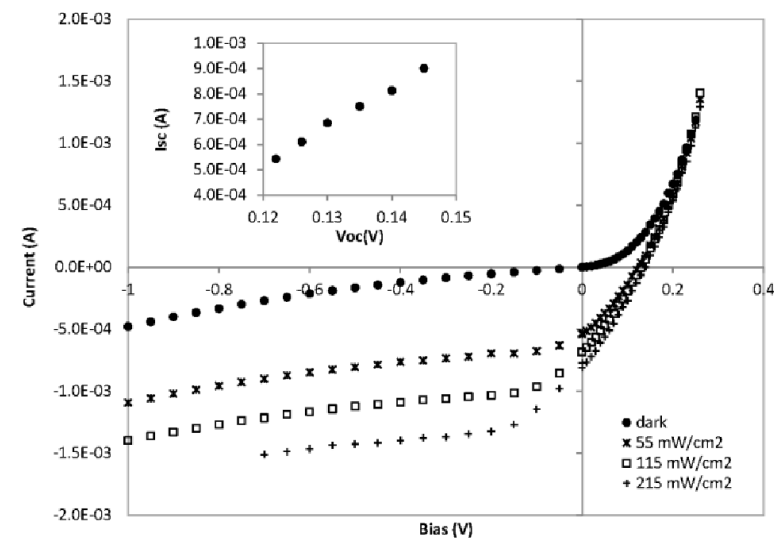

Fig. 3. Room temperature $I-V$ characteristics under dark and at various illumination intensities. In the inset: short circuit current vs open circuit voltage.

Illuminating the photovoltaic cell with optical radiation shifts the I-V curves by the amount of photocurrent $I_{\mathrm{p}}$.

$$
I=I_{0} \exp (q V / \beta k T)-I_{\mathrm{p}},
$$

where $I_{\mathrm{p}}$ is the photocurrent. When a photovoltaic cell is illuminated, the current-voltage curve is shifted to the fourth quadrant [1], giving open circuit voltage $\left(V_{\mathrm{oc}}\right)$ and short circuit current $\left(I_{\mathrm{sc}}\right)$. The inset of Fig. 3 shows $I_{\mathrm{Sc}}$ versus $V_{\mathrm{oc}}$ values of the structure as a function of illumination intensity. It can be seen from the figure that, as expected from the ideal diode model, $I_{\mathrm{sc}}$ increases with the increasing $V_{\text {oc }}$.
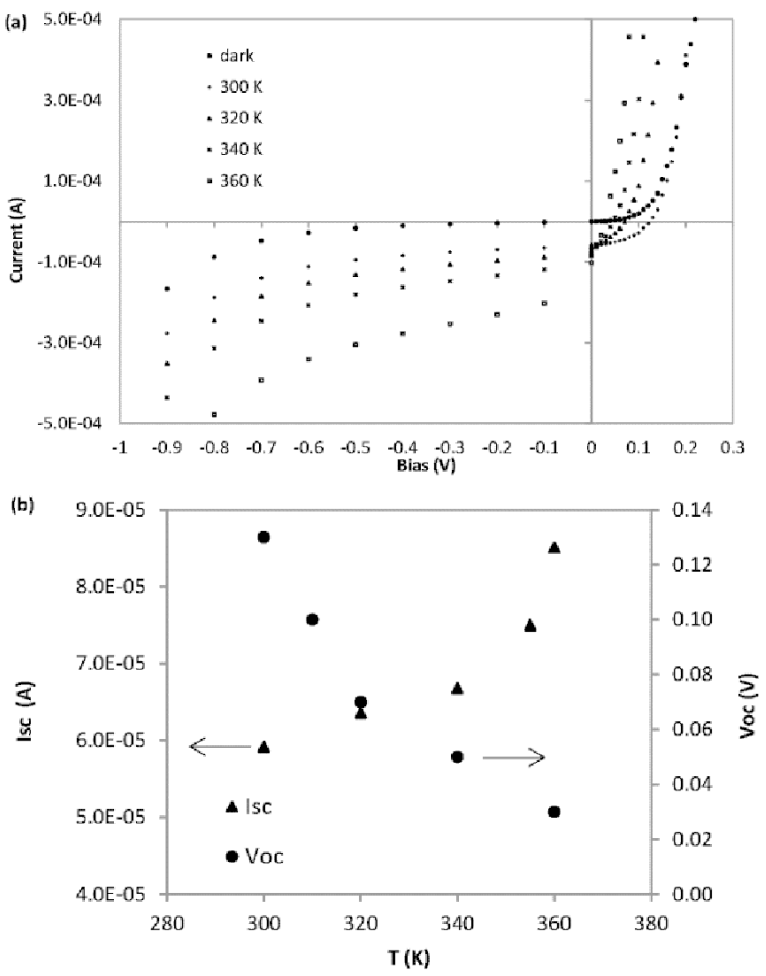

Fig. 4. Dependences of a) photocurrent b) short circuit current and open circuit voltage on temperature. 
Figures $4 \mathrm{a}$ and $4 \mathrm{~b}$ show temperature dependences of photocurrent and photovoltaic parameters of the investigated structure under the fixed radiation intensity. The bandgap width decreases with increasing temperature of the structure. Thus, reverse saturation current increases, which causes a reduction in the $V_{\mathrm{oc}}$ and the production of carriers becomes increasingly significant, which implies a weak increase in the $I_{\mathrm{sc}}[15]$. The results demonstrate that $I_{\mathrm{sc}}$ increases, while $V_{\mathrm{oc}}$ decreases with the increasing temperature as expected.

\section{Conclusions}

p-GaSb/p-GaInAsSb/n-GaAlAsSb thermophotovoltaic diode with an active area of $1 \mathrm{~mm}$ in diameter has been investigated. Dark current mechanisms of the structure were explained for both forward and reverse regions in the temperature range of $100-345 \mathrm{~K}$. In addition, the current-voltage characteristics were analyzed under different illumination intensities. The photovoltaic parameters such as short circuit current and open circuit voltage were investigated at varying temperature.

\section{References}

[1] T. Bauer, Thermophotovoltaics - Basic Principles and Critical Aspects of System Design, Springer, 2011.

[2] R.E. Nelson, Semicond. Sci. Technol. 18, 141 (2003).

[3] V.P. Khvostikov, S.V. Sorokina, N.S. Potapovich, O.A. Khvostikova, A.V. Malievskaya, A.S. Vlasov, M.Z. Shvarts, N.Kh. Timoshina, V.M. Andreev, Semiconductors. 44, 255 (2010).
[4] M. Ahmetoglu, B. Kucur, I.A. Andreev, E.V. Kunitsyna, M.P. Mikhailova, Yu.P. Yakovlev, Infrared Phys. Techn. 53, 399 (2010).

[5] M.H.M. Reddy, J.T. Olesberg, C. Cao, J.P. Prineas, Semicond. Sci. Technol. 21, 267 (2006).

[6] M.G. Mauk, V.M. Andreev, Semicond. Sci. Technol. 18, S191 (2003).

[7] K. Qiu, A.C.S. Hayden, Appl. Energy. 91, 304 (2012).

[8] M.P. Mikhailova, A.N. Titkov, Semicond. Sci. Technol. 9, 1279 (1994).

[9] Y. Wang, N.F. Chen, X.W. Zhang, T.M. Huang, Z.G. Yin, Y.M. Bai, Semicond. Sci. Technol. 25, 095002 (2010).

[10] V.M. Andreev, Next Generation Photovoltaics, Ed. A. Marti, A. Luque, Inst. Phys. Publish, U.K. 2004

[11] B. Kucur, M. Ahmetoglu, I.A. Andreev, E.V. Kunitsyna, M.P. Mikhailova, Y.P. Yakovlev, Acta Phys. Pol. A 125, 411 (2014).

[12] E.V. Kunitsyna, I.A. Andreev, M.P. Mikhailova, Ya.A. Parkhomenko, Yu.P. Yakovlev, Proceedings of SPIE., 2000, p. 4340.

[13] S.M. Sze, Physics of Semiconductor Devices Physics and Technology, Wiley, New York, 1985.

[14] J.L. Moll, Physics of Semiconductors, McGraw Hill, New York, 1964.

[15] F. Bouzid, L. Dehimi, Rev. Energies Renouvelables 15, 383 (2012). 